\title{
Alteraciones metabólicas sutiles en adolescentes con obesidad y síndrome de ovario poliquístico
}

\author{
Víctor Saúl Vital-Reyes¹, Mardia Guadalupe Lopez-Alarcón², Patricia Inda-Icaza ${ }^{3}$ \\ y Concepción Márquez-Maldonado 3 \\ ${ }^{1}$ Departamento de Biología de la Reproducción y Gineco-endocrinología, Unidad Médica de Alta Especialidad (UMAE) Hospital de Ginecología y \\ Obstetricia 3 Dr. Víctor Manuel Espinoza de los Reyes Sánchez, Centro Médico Nacional La Raza, Instituto Mexicano del Seguro Social (IMSS); \\ ${ }^{2}$ Unidad de Investigación Médica en Nutrición Humana, UMAE Hospital de Pediatría, Centro Médico Nacional Siglo XXI, IMSS; ${ }^{3}$ Escuela de Nutrición, \\ Universidad Anáhuac Campus Norte, Ciudad de México
}

\section{Resumen}

Objetivo: Determinar algunas alteraciones metabólicas sutiles en adolescentes con obesidad y síndrome de ovario poliquístico (SOP). Material y métodos: Se efectuó un estudio clínico transversal comparativo en un grupo de adolescentes con obesidad y caracterizadas con SOP acorde a los criterios del Consenso de Rotterdam. Se realizó historia clínica, antropometría y ultrasonido pélvico ginecológico con el fin de determinar los volúmenes ováricos, el número de folículos antrales y el grosor endometrial, así como la determinación sanguínea de glucosa, insulina, lipoproteínas, interleucina-6, factor de necrosis tumoral alfa, testosterona, dehidroepiandrosterona, globulina transportadora de hormonas sexuales, leptina, adiponectina, factor de crecimiento parecido a la insulina-1 y determinación del índice de andrógenos libre, testosterona libre y biodisponible, así como la resistencia a la insulina a través del HOMA (homeostasis model assessment). Se realizó estadística descriptiva, análisis comparativo con $t$ de Student o con $U$ de Mann Whitney, de correlación bivariada con Pearson y de regresión múltiple. Resultados: De 180 adolescentes, se estudiaron 47 que cumplieron los criterios de inclusión. El promedio de edad fue de 13.5 años. La Z-score del índice de masa corporal (IMC) para el percentil 95 tuvo una media de 2.5. El 80\% de las adolescentes estudiadas tuvo distribución central de la grasa. En el 95\% se documentó hiperinsulinismo. Las dislipidemias más frecuentes fueron hipertrigliceridemia en el 57\% e hipercolesterolemia en el 12.8\%. El 25.5\% de las adolescentes presentó dos de los tres criterios diagnósticos vigentes para SOP. Las pacientes con SOP tuvieron concentraciones séricas de glucosa, insulina y enzimas hepáticas mayores que las que no tenían SOP. La testosterona libre se asoció positivamente con el IMC y las concentraciones de insulina, pero la magnitud de la asociación fue mayor en las pacientes con SOP. Conclusiones: Las alteraciones metabólicas encontradas en las adolescentes con obesidad y SOP sugieren que las manifestaciones clínicas del SOP no solo forman parte de una endocrinopatía que aparece con frecuencia en la edad reproductiva, sino que también caracterizan al SOP como una enfermedad metabólica que conlleva riesgos para la salud a corto, mediano y largo plazo en adolescentes obesas, y que ameritan de acciones de intervención en la prevención, el diagnóstico integral y el tratamiento oportuno con el fin de limitar el daño en la evolución natural del SOP.

PALABRAS CLAVE: Adolescentes. Síndrome de ovario poliquístico. Obesidad.

Correspondencia:

Víctor Saúl Vital-Reyes

E-mail: victor.vital@imss.gob.mx
Fecha de recepción: 07-08-2017

Fecha de aceptación: 07-09-2017

DOI://dx.doi.org/10.24875/GMM.M17000005
Gac Med Mex. 2017;153;Sup 2:S34-S41 Contents available at PubMed www.anmm.org.mx 


\section{Abstract}

Objective: To evaluate the frequency of some subtle metabolic alterations in a group of adolescents with obesity and polycystic ovary syndrome (PCOS). Materials and Methods: A cross-sectional, comparative study was conducted in a group of adolescents with obesity, and characterized as with or without PCOS according with the Rotterdam Consensus. Medical history, anthropometry, gynecologic pelvic ultrasound (to evaluate ovarian volumes, number of antral follicles and endometrial width), as well as serum glucose, insulin, lipoproteins, interleukin-6, tumor necrosis factor alpha, total testosterone, dehydroepiandrosterone, sexual hormones binding globulin, leptin, adiponectin and insulin-like growth factor 1 , the free-androgen index, free and available testosterone, and homeostatic model assessment index were calculated. For statistics, mean and standard deviation, or median and ranges were used for description as appropriate. Likewise, Student t-test or Mann-Whitney test were used for comparisons. Results: From a sample of 180 adolescents, 47 attached to selection criteria. Mean age was 13.5 year and Z-score 2.5. Eighty percent of adolescents presented central distribution of body fat and 95\% hyperinsulinemia. The more frequent dyslipidemias were hypertriglyceridemia in 57\% and hypercholesterolemia in $12.8 \%$; $25.5 \%$ of adolescents presented two out of three criteria for polycystic ovary syndrome (PCOS). Body mass index and insulin were correlated with free testosterone, but the multivariate analysis demonstrated that the magnitude of the association was significantly higher in SOP patients. Conclusions: The metabolic alterations detected in obese adolescents with SOP suggest that the clinical manifestations that accompany the syndrome characterize the PCOS as a metabolic disease, which carry important health risks at short, medium and long term. Therefore, they merit intervening actions to prevent, diagnose and provide timing treatment in order to limit the damage in the course of the natural history of PCOS.

KEY WORDS: Adolescents. Polycystic ovary syndrome. Obesity.

\section{Introducción}

El síndrome de ovario poliquístico (SOP) es un trastorno endocrino-metabólico multifactorial heterogéneo, que con frecuencia se manifiesta en la edad reproductiva. Además, se ha documentado que el SOP afecta de igual manera a las adolescentes. En general, la prevalencia del SOP es del 6 al 10\%, y parece ser que en pacientes con obesidad esta prevalencia aumenta de forma considerable. Los criterios diagnósticos del SOP se basan en consensos, donde se resaltan las alteraciones menstruales (oligo-amenorrea), el hiperandrogenismo clínico (hirsutismo, acné, alopecia) o bioquímico (concentraciones séricas elevadas de andrógenos), y la morfología ultrasonográfica de ovarios poliquísticos ${ }^{1}$.

A pesar de que la obesidad no ha sido considerada un criterio diagnóstico de SOP, esta se presenta en alrededor del $30 \%$ de las pacientes en edad reproductiva con SOP, y se ha reportado que un porcentaje importante de mujeres en edad reproductiva con SOP, aunado a las alteraciones hormonales propias del SOP, presentan alteraciones metabolicas ${ }^{2}$.

De la misma manera, en pacientes con SOP se ha reportado un incremento del riesgo cardio-metaboli$\mathrm{CO}^{3}$, y el SOP ha sido considerado un factor de riesgo importante de desarrollo de cáncer endometrial ${ }^{4}$.
La categorización clínica del SOP es heterogénea. Se ha reportado que el hiperandrogenismo clínico o bioquímico se observa en alrededor del $70 \%$ de las pacientes, mientras que el $27 \%$ presenta disfunción ovulatoria y en alrededor del $20 \%$ de las pacientes con SOP se documenta la presencia de ovarios de aspecto poliquístico por ultrasonografía acorde con los criterios vigentes, y la prevalencia de resistencia a la insulina (RI) es variable ${ }^{5}$.

La interacción del exceso de andrógenos, el alto índice de masa corporal (IMC) y la RI en pacientes con SOP forma parte de la fisiopatología de este síndrome. El hiperandrogenismo y la RI en pacientes con SOP coexisten en la regulación de la esteroidogénesis ovárica, así como en la regulación de la síntesis de SHGB (globulina fijadora de hormonas sexuales) y de IFG-1 (factor de crecimiento parecido a la insulina tipo 1). ${ }^{6}$

Hickey, et al. ${ }^{7}$ publicaron en el año 2011 que, en un grupo de adolescentes con promedio de edad de 15.2 años, el $51 \%$ presentó irregularidades menstruales, hirsutismo el $3.5 \%$ y ovarios de aspecto poliquístico el $35 \%$, con lo cual se integró el diagnóstico de SOP de acuerdo con los criterios de Rotterdam en el $18.5 \%$ de las adolescentes.

El sobrepeso y la obesidad tienen una alta prevalencia en las pacientes con SOP, lo que incrementa el riesgo de complicaciones como infertilidad, sangrado uterino anormal, intolerancia a los hidratos de 
carbono, dislipidemia, síndrome metabólico y cáncer ginecológico. A menudo no es considerado el diagnóstico de SOP en la adolescente, debido principalmente a que las alteraciones ginecológicas se asocian a adaptaciones propias de la etapa del desarrollo que están cursando. Desde este contexto, la meta del presente trabajo fue determinar las alteraciones metabólicas sutiles en este grupo vulnerable de riesgo.

\section{Métodos}

Se realizó un estudio clínico transversal comparativo en una población de adolescentes atendidas en las Unidades de Medicina Familiar número 1, 10 y 26 del Instituto Mexicano del Seguro Social en la Ciudad de México. Fueron incluidas niñas de edad comprendida entre 12 y 18 años, con IMC por arriba del percentil 95 de la National Center for Health Statistics (NCHS), para someterse a ultrasonido pélvico y toma de muestra sanguínea de una vena periférica. Todas las pacientes aceptaron participar voluntariamente a través de asentimiento informado y carta de consentimiento autorizada por los padres o tutores. El estudio fue autorizado por el Comité de Investigación, con número de registro CNIC-2011-785-037.

La caracterización diagnóstica de SOP fue acorde con los criterios de Rotterdam, y todas las pacientes incluidas en el grupo de SOP presentaron por lo menos dos de los tres siguientes criterios: alteraciones menstruales, hiperandrogenismo clínico o bioquímico, o morfología ultrasonográfica de poliquistosis ovárica ( $\geq 12$ folículos ováricos de 2-9 mm) .

La obesidad, definida como una acumulación anormal o excesiva de grasa, fue determinada a través del IMC. Los valores obtenidos de IMC se convirtieron para su análisis en Z-score utilizando las tablas de la NCHS como referencia?.

Acorde al diagnóstico de SOP, se formaron dos grupos de estudio: adolescentes con obesidad y SOP, $y$ adolescentes obesas sin SOP.

En todas las pacientes incluidas en este estudio se efectuaron historia clínica, evaluación del hirsutismo mediante la escala de Ferriman-Gallwey, antropometría, biometría hemática completa, pruebas de coagulación, glucosa, urea, creatinina, perfil de lípidos, pruebas de función hepática, testosterona, insulina, hormona estimulante del tiroides, dehidroepiandrosterona (DHEA), IGF-1, SHBG, proteína C reactiva (PCR), interleucina-6 (IL-6), factor de necrosis tumoral alfa (TNF- $\alpha$ ) y ultrasonido pélvico ginecológico con el fin de determinar los volúmenes ováricos, el número y el diámetro de folículos ováricos, y el grosor endometrial.

La antropometría se realizó por personal estandarizado y se determinaron el peso, la estatura y las circunferencias de cintura y cadera, utilizando una báscula de pedestal con estadiómetro y cinta métrica no elástica, respectivamente. Se calculó el IMC dividiendo el peso en kilos entre el cuadrado de la estatura en metros, según la fórmula: $I M C=$ peso $(\mathrm{kg}) /$ estatura $\left(\mathrm{m}^{2}\right)^{10}$.

El ultrasonido pélvico se realizó por personal estandarizado utilizando un equipo marca Samsung Medison sonoace R3 con transductor lineal de 7.5 a $13 \mathrm{MHz}$, y se determinaron los volúmenes ováricos, el número y el tamaño de folículos preantrales, y el grosor endometrial en condiciones basales.

El perfil lipídico incluyó la determinación de colesterol de lipoproteínas de baja densidad (LDL-C), colesterol de lipoproteínas de alta densidad (HDL-C) y triglicéridos. Los puntos de corte de las concentraciones séricas fueron $<200 \mathrm{mg} / \mathrm{dl}$ para colesterol total, $>52 \mathrm{mg} / \mathrm{dl}$ para HDL-C y $<150 \mathrm{mg} / \mathrm{dl}$ para triglicéridos.

La RI fue evaluada a través del índice de HOMA (homeostasis model assessment) propuesto por Matthews, et al. ${ }^{11}$. El HOMA es sensible para identificar niños y adolescentes con RI, pero la información sobre los puntos de corte es muy variable, por lo que utilizamos los propuestos por Keskin, et al. ${ }^{12}$ para adolescentes (3.16).

Las determinaciones de testosterona total (TT) y de SBHG se realizaron por quimioluminiscencia utilizando estuches comerciales. Con los valores de las concentraciones de TT y SHBG se determinaron la testosterona libre $(T L)$ y la testosterona biodisponible (TB) de acuerdo con las siguientes fórmulas:

$\mathrm{TB}(\mathrm{mol} / \mathrm{l})=\{(\mathrm{ka} \times[$ albúmina $] \times[\mathrm{TL}]) /(1+\mathrm{ka} \times[\mathrm{TL}])\}$ $+[\mathrm{TL}]$

$\operatorname{TL}(\mathrm{mol} / \mathrm{l})=\left\{-\mathrm{b}+\sqrt{ }\left(\mathrm{b}^{2}+4 \mathrm{a}\left[\mathrm{TT}^{2}\right]\right)\right\} / 2 \mathrm{a}$ donde:

$\mathrm{a}=\mathrm{ka}+\mathrm{kt}+(\mathrm{ka} \times \mathrm{kt}) \times([\mathrm{SHBG}]+[$ albúmina $]$ $-[\mathrm{TT}])$

$\mathrm{b}=1+\mathrm{kt} \times[\mathrm{SHBG}]+\mathrm{ka} \times$ [albúmina $]-(\mathrm{ka}+\mathrm{kt}) \times$ [TT]

Las constantes de afinidad de unión de la testosterona a la SHBG (kt) y la albúmina (ka) son: $10 \times 10^{8} \mathrm{I} / \mathrm{mol}$ y $3,6 \times 10^{4} \mathrm{I} / \mathrm{mol}$, respectivamente.

Para la determinación del colesterol total, las lipoproteínas y los triglicéridos se utilizó un método colorimétrico enzimático.

Para el análisis estadístico se calcularon las frecuencias absolutas y relativas, medidas de tendencia 
central y de dispersión acorde con el tipo de distribución de cada variable. La contrastación estadística de variables numéricas continuas se realizó utilizando la t de Student o la U de Mann Whitney, dependiendo de la distribución de la variable. Se estableció un nivel de significancia de $p<0.05$. Para el manejo de los datos se utilizó el paquete estadístico SPSS v16.

\section{Resultados}

De las 180 adolescentes convocadas al estudio, fueron seleccionadas 47 que reunieron los criterios de inclusión. La edad promedio del grupo estudiado fue de $13.5 \pm 2$ años, con un rango de 11 años. El IMC fue de $31 \pm 3$ y la Z-score fue de $2.5 \pm 0.4$. La circunferencia de la cintura fue de $96 \pm 9 \mathrm{~cm}$, y el desarrollo sexual valorado a través de Tanner fue


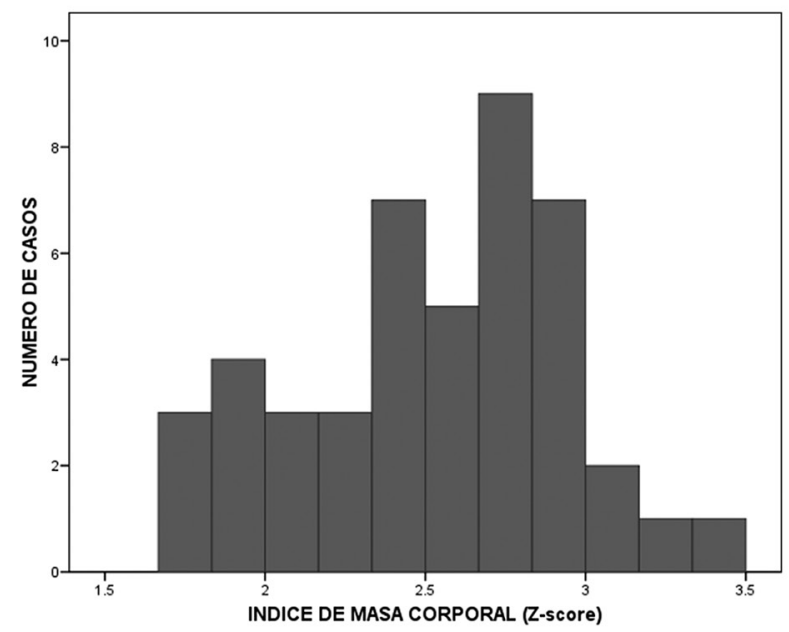

$3.9 \pm 0.8$. La distribución de estas variables se muestra en la figura 1.

La aparición de la menarca tuvo un rango de 9 a 13 años y un promedio de $11.1 \pm 1$. El hirsutismo evaluado con la escala de Ferriman Galwey tuvo una puntuación promedio de $4.6 \pm 2.2$, con una amplitud de 2 a 12. Las concentraciones basales de insulina expresadas en $\mu \mathrm{UI} /$ dl tuvieron un promedio de $33 \pm 17$ y una amplitud de 11 a 119. La RI evaluada a través de HOMA tuvo una amplitud de 2 a 31 , con un promedio de $7 \pm 4$. La distribución de estas variables se muestra en la figura 2.

El $80.9 \%(n=38)$ de las adolescentes estudiadas tuvo distribución central de la grasa corporal, evaluada mediante la circunferencia de la cintura. El 95.7\% ( $n=45)$ de las adolescentes estudiadas mostró concentraciones basales de insulina sérica superiores a $15 \mu \mathrm{UI} / \mathrm{dl}$. Con respecto a la dislipidemia, el $57.4 \%$ ( $n=27$ ) presentó triglicéridos superiores a $150 \mathrm{mg} / \mathrm{dl}$

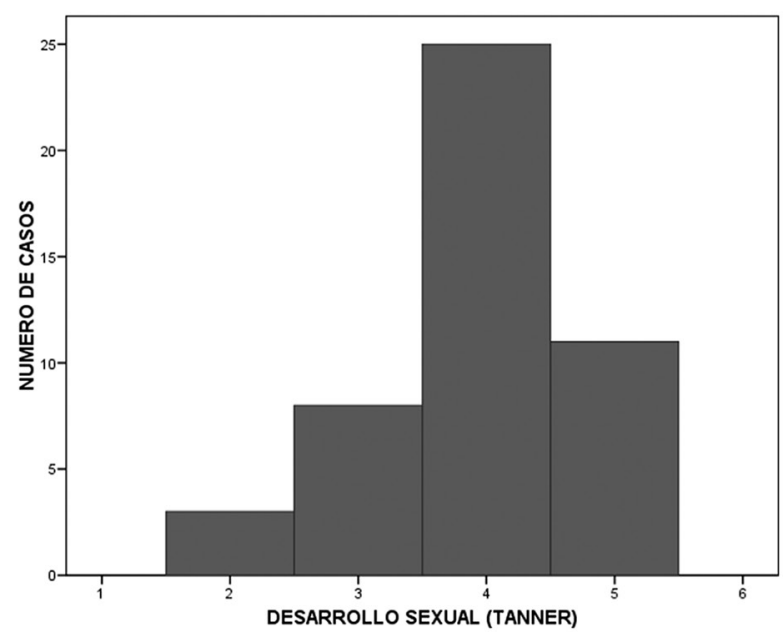

Figura 1. Distribución de edad, Z-score del IMC, circunferencia de cintura y desarrollo sexual en un grupo de adolescentes con obesidad $(n=47)$. 

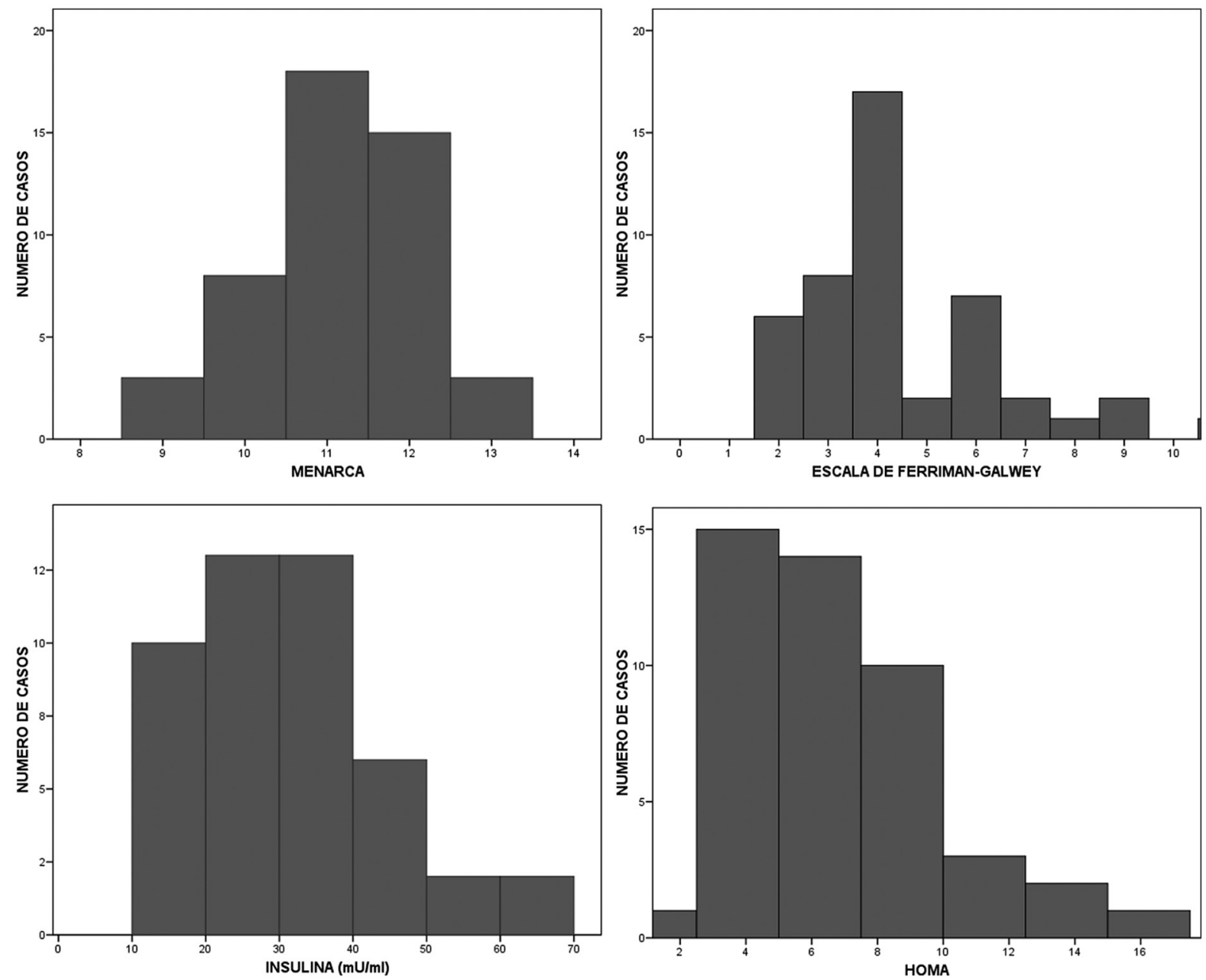

Figura 2. Distribución de la menarca (escala de Ferriman-Galwey), insulina sérica y HOMA $(n=47)$.

y el $12.8 \%(n=6)$ hipercolesterolemia. Con respecto al funcionamiento hepático, en el $23.4 \%(n=11)$ y el $48.09 \%(n=23)$ se encontró elevación de alanina aminotransferasa (TGP) y aspartato aminotransferasa (TGO), respectivamente.

De las 47 adolescentes que cumplieron con los criterios de selección, 12 (25.53\%) cumplieron con los criterios para SOP. Las variables edad, IMC, Z-score del IMC, cintura, Tanner, glucosa, insulina, HOMA, colesterol, VLDL, HDL, LDL, triglicéridos, TGP, TGO, gama glutamil transpeptidasa (GGT), TNF- $\alpha$, PCR, leptina, adiponectina, Ferriman-Galwey, volúmenes ováricos, grosor endometrial, DHEA, SHBG, testosterona, índice de andrógenos libres, TL, porcentaje de TL, TB, porcentaje de TB e IGF-1 se presentan en la tabla 1 estratificadas por grupos de estudio. En esta tabla se muestra que, además de las variables asociadas con el SOP (TL, SHBG y volúmenes ováricos), las pacientes con SOP presentaron mayor IMC y mayores concentraciones de glucosa, insulina y enzimas hepáticas comparadas con las adolescentes que no presentaron SOP.

El análisis de correlación bivariada demostró una asociación positiva de la TL con el IMC ( $r=039$; $p=0008)$ y con las concentraciones de insulina $(r=036 ; p=0015)$, pero negativa con la SHBG $(r=-097 ; p<001)$. El análisis de regresión múltiple demostró que la magnitud de la asociación entre estas variables fue mayor en las pacientes con SOP (Fig. 3).

\section{Discusión}

Las alteraciones clínicas que conlleva el SOP a lo largo de la vida reproductiva de la mujer representan causas frecuentes de atención médica.

La prevalencia de SOP en la población general es del 6 al 10\%. En la población de nuestro estudio, conformada por adolescentes con obesidad, encontramos 


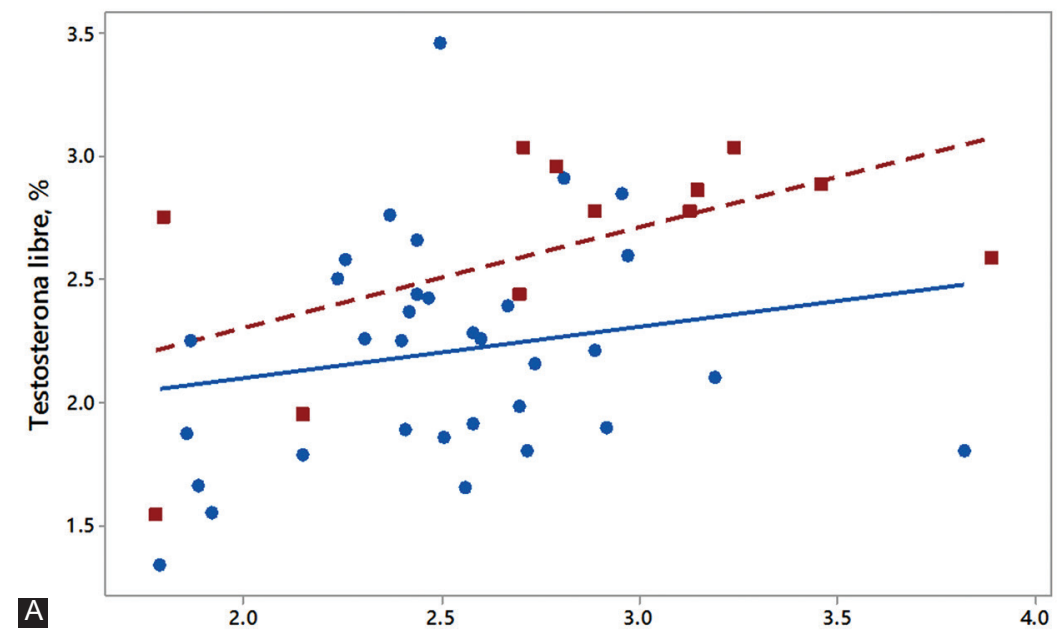

IMC, Z-score

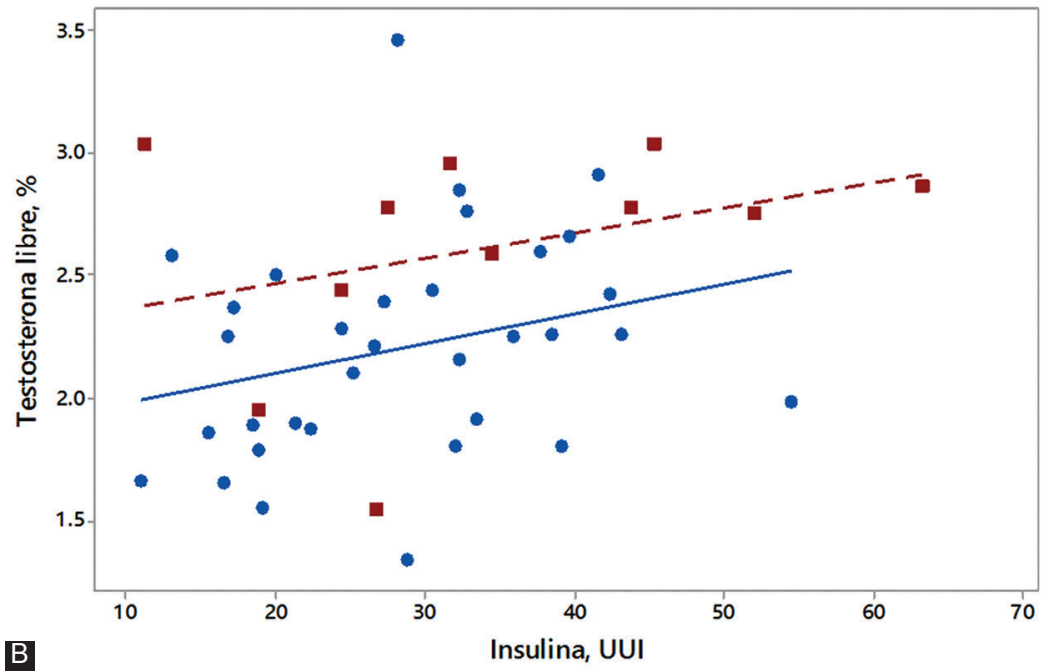

Figura 3. Asociación entre la TL y el IMC (A) y la concentración de insulina (B), por grupos de estudio. Las líneas rojas corresponden a las pacientes con SOP. Valor para $A: R^{2}=0.25$ y $p=0.028$. Valor para $B: R^{2}=0.22$ y $p=0.03$.

Tabla 1. Análisis comparativo de algunos parámetros clínicos y paraclínicos de adolescentes obesas con y sin SOP

\begin{tabular}{lccc}
\hline Variable & $\begin{array}{c}\text { Adolescentes } \sin \text { SOP } \\
\mathbf{n}=\mathbf{3 5}\end{array}$ & $\begin{array}{c}\text { Adolescentes con SOP } \\
\mathbf{n}=\mathbf{1 2}\end{array}$ & $\mathbf{p}^{*}$ \\
\hline Edad (años) & $13.5 \pm 2.1$ & $13.6 \pm 1.4$ & 0.79 \\
IMC & $30.6 \pm 3.04$ & $33.1 \pm 4.2$ & 0.04 \\
Z-score & $2.5 \pm 0.42$ & $2.8 \pm 0.64$ & 0.08 \\
Cintura $(\mathrm{cm})$ & $95.2 \pm 8.9$ & $100.3 \pm 13$ & 0.11 \\
Tanner & $3.8 \pm 0.8$ & $4.2 \pm 0.4$ & 0.41 \\
Glucosa (mg/dl) & $86.9 \pm 8.7$ & $92 \pm 9.4$ & 0.06 \\
Insulina $(\mu \mathrm{U} / \mathrm{dl})$ & $28.4 \pm 10.3$ & $41.6 \pm 28.5$ & 0.07 \\
HOMA & $6.1 \pm 2.4$ & $9.7 \pm 7.6$ & 0.07 \\
Colesterol $(\mathrm{mg} / \mathrm{dl})$ & $169 \pm 27$ & $172 \pm 37$ & 0.84 \\
VLDL (mg/dl) & $31 \pm 20$ & $28 \pm 15$ & 0.62 \\
\hline
\end{tabular}


Tabla 1. Análisis comparativo de algunos parámetros clínicos y paraclínicos de adolescentes obesas con y sin SOP (Continued)

\begin{tabular}{|c|c|c|c|}
\hline Variable & $\begin{array}{l}\text { Adolescentes sin SOP } \\
\qquad \mathrm{n}=35\end{array}$ & $\begin{array}{l}\text { Adolescentes con SOP } \\
\qquad n=12\end{array}$ & $\mathbf{p}^{*}$ \\
\hline $\mathrm{HDL}(\mathrm{mg} / \mathrm{dl})$ & $49 \pm 8$ & $49 \pm 4$ & 0.69 \\
\hline $\mathrm{LDL}(\mathrm{mg} / \mathrm{dl})$ & $89 \pm 21$ & $93 \pm 27$ & 0.62 \\
\hline Triglicéridos (mg/dl) & $155 \pm 103$ & $141 \pm 75$ & 0.62 \\
\hline TGP (UI/I) & $23.9 \pm 7.2$ & $41.2 \pm 27.4$ & 0.03 \\
\hline TGO (UI/l) & $32.4 \pm 7.5$ & $49.6 \pm 35$ & 0.06 \\
\hline GGT (UI/I) & $16.1 \pm 7.9$ & $26.5 \pm 8.1$ & 0.04 \\
\hline IL-6 (pg/ml) & $1.8 \pm 1.4$ & $2.6 \pm 2.7$ & 0.36 \\
\hline TNF- $\alpha(p g / m l)$ & $2.2 \pm 1.3$ & $1.7 \pm 0.8$ & 0.11 \\
\hline PCR (mg/ml) & $2.7 \pm 3.4$ & $4.7 \pm 5.3$ & 0.24 \\
\hline Leptina (ng/ml) & $41 \pm 13$ & $44 \pm 21$ & 0.63 \\
\hline Adiponectina (ng/ml) & $5.9 \pm 3$ & $6.5 \pm 2.8$ & 0.50 \\
\hline Ferriman-Galwey & $4.3 \pm 1.7$ & $5.6 \pm 3.2$ & 0.19 \\
\hline Volumen ovario derecho (cc) & $7.1 \pm 2.1$ & $9.2 \pm 2.8$ & 0.04 \\
\hline Volumen ovario izquierdo (cc) & $7.4 \pm 2.4$ & $9.0 \pm 2.7$ & 0.09 \\
\hline Endometrio (mn) & $6.7 \pm 2.9$ & $7.6 \pm 2.8$ & 0.34 \\
\hline DHEA $(\mu \mathrm{g} / \mathrm{dl})$ & $132 \pm 96$ & $199 \pm 108$ & 0.04 \\
\hline SHBG (nmoles/l) & $24.2 \pm 9.8$ & $16.3 \pm 9.6$ & 0.01 \\
\hline Testosterona (ng/dl) & $22.3 \pm 18$ & $23.0 \pm 11$ & 0.44 \\
\hline Índice de andrógenos libres (\%) & $3.1 \pm 2.5$ & $5.2 \pm 3.2$ & 0.05 \\
\hline $\mathrm{TL}(\mathrm{ng} / \mathrm{dl})$ & $0.41 \pm 0.3$ & $0.62 \pm 0.3$ & 0.04 \\
\hline TL \% & $2.2 \pm 0.4$ & $2.6 \pm 0.5$ & 0.006 \\
\hline $\mathrm{TB}(\mathrm{ng} / \mathrm{dl})$ & $9.4 \pm 7.3$ & $14.6 \pm 7.6$ & 0.04 \\
\hline TB \% & $51 \pm 10$ & $62 \pm 11$ & 0.004 \\
\hline IGF-1 (ng/ml) & $453 \pm 151$ & $406 \pm 124$ & 0.29 \\
\hline
\end{tabular}

*La comparación estadística se realizó mediante t de Student o U de Mann Whitney, según la distribución de los datos.

Las cifras representan el promedio \pm desviación estándar de las variables estudiadas.

que, acorde con los criterios vigentes, en el $25.5 \%$ se documentó el diagnóstico de SOP. La proporción encontrada está por arriba de lo reportado por Hickey, et al. ${ }^{7}$ en 2011 , que acorde también con los criterios de Rotterdam encontraron una proporción de un $18.5 \%$ de las adolescentes. Sin embargo, debemos destacar que un sesgo de selección, que explicaría una mayor proporción de SOP en nuestras adolescentes estudiadas, podría ser que incluimos solo adolescentes con obesidad. Aunque la obesidad no ha sido considerada un criterio diagnóstico de SOP, se estima que cerca del $50 \%$ de las pacientes con SOP son obesas, y se ha calculado que en poblaciones de riesgo, en las que la prevalencia de obesidad es mayor ( $37.5 \%$ en mujeres mexicanas adultas, según datos de la ENSANUT 2012), ${ }^{13}$ hasta el $65 \%$ de las pacientes con SOP cursan con obesidad ${ }^{14,15}$. De la misma manera, se ha observado que en mujeres méxico-americanas la prevalencia global de SOP aumenta hasta el $13 \%^{16}$.

La obesidad característica del SOP tiene un patrón de acumulación de grasa predominantemente visceral. Nosotros encontramos que el $80 \%$ de las adolescentes estudiadas tiene distribución central de la grasa, y que en el $95 \%$ de estas pacientes se documenta hiperinsulinismo tomando un punto de corte de insulina basal $\geq 15 \mu \mathrm{UI} / \mathrm{ml}$. La participación de la RI 
y del hiperinsulinismo en la fisiopatología del SOP ha sido previamente documentada, y en la RI se incrementa la síntesis ovárica y suprarrenal de andrógenos, se disminuye la producción de SHGB y se altera la liberación hipofisaria de gonadotrofinas ${ }^{17}$.

La obesidad se ha considerado un factor de riesgo metabólico, pero de manera independiente la distribución central de la grasa incrementa el riesgo de dislipidemia $^{18}$. Nosotros encontramos que el $57 \%$ de las adolescentes estudiadas mostraba triglicéridos mayores de $150 \mathrm{mg} / \mathrm{ml}$ y el $13 \%$ presentó hipercolesterolemia.

Cuando comparamos las diferentes variables estudiadas, entre adolescentes obesas con y sin SOP, solo encontramos diferencias estadísticamente significativas en aquellas variables que son elementos diagnósticos del SOP, como son el hiperandrogenismo, las alteraciones menstruales y la disfunción ovulatoria. Resultado que podría ser inherente al sesgo de caracterización del SOP o la heterogeneidad de la presentación clínica del SOP que ya ha sido descrita previamente'.

Es importante señalar que una de las principales limitaciones metodológicas del presente estudio es el tamaño de la población estudiada, y posiblemente el sesgo de selección de la obesidad como criterio de inclusión.

En todo el mundo, alrededor de 1.6 billones de adultos tienen sobrepeso y más de 400 millones presentan obesidad. Este problema involucra a la población pediátrica, a tal grado que la obesidad infantil es uno de los problemas de salud pública más serios del siglo xxi porque la prevalencia de sobrepeso y obesidad en el niño se ha incrementado a una velocidad alarmante. En México, la prevalencia de sobrepeso y obesidad combinadas para preescolares, escolares y adolescentes fue de aproximadamente el 15, el 26 y el $31 \%$, respectivamente, en el año 2006; estas cifras representan el doble de las reportadas en la Encuesta Nacional de Nutrición publicada en el año 2012. En Ciudad de México, actualmente, las prevalencias de sobrepeso y obesidad combinadas se encuentran en el rango del 17.8 al $21.0 \%$ y del 41 al $43 \%$ para escolares y adolescentes de las cuatro delegaciones del Instituto Mexicano del Seguro Social. Desde este contexto, las alteraciones metabólicas sutiles encontradas en adolescentes con obesidad y reportadas en el presente trabajo, y previamente publicadas por otros autores en mujeres con $\mathrm{SOP}^{19}$, sugieren que las manifestaciones clínicas del SOP no solo forman parte de una endocrinopatía que aparece con frecuencia en la edad reproductiva, sino que también existen alteraciones sutiles que caracterizan al SOP como parte de una enfermedad metabólica que conlleva riesgos para la salud a corto, mediano y largo plazo en adolescentes obesas, y que ameritan de acciones de intervención en prevención, diagnóstico integral y tratamiento oportuno, con el fin de limitar el daño en la evolución natural del SOP.

\section{Bibliografía}

1. Azziz R, Carmina E, Dewailly D, et al. Task Force on the Phenotype of the Polycystic Ovary Syndrome of The Androgen Excess and PCOS Society. The androgen excess and PCOS Society criteria for the polycystic ovary syndrome: the complete task force report. Fertil Steril. 2009;91:456-88.

2. Kumar AN, Naidu JN, Satyanarayana U, et al. Metabolic and endocrine characteristics of Indian women with polycystic ovary syndrome. Fertil Steril. 2016;10:22-8.

3. Talbott EO, Zborowski JV, Rager JR, et al. Evidence for an association between metabolic cardiovascular syndrome and coronary and aortic calcification among women with polycystic ovary syndrome. End Met. 2004;89:5454-61.

4. Fearnley EJ, Marquart L, Spurdle AB, et al.; Australian Ovarian Cancer Study Group and Australian National Endometrial Cancer Study Group. Polycystic ovary syndrome increases the risk of endometrial cancer in women aged less than 50 years: an Australian case-control study. Cancer Causes Control. 2010;21:2303-8.

5. Barber TM, Dimitriadis GK, Andreou A, et al. Polycystic ovary syndrome: insight into pathogenesis and a common association with insulin resistance. Clin Med. 2015;15:72-6.

6. De la Parra I, Cesaratto I, Flamini K. En el síndrome de ovario poliquístico, ¿cuál es la asociación entre insulino resistencia, menarca, ciclo menstrual, índice de masa corporal, hiperandrogenismo y bulimia nerviosa? Rev Endocr Gin y Repr. 2011;13:53-67.

7. Hickey M, Doherty DA, Atkinson $\mathrm{H}$, et al. Clinical, ultrasound and biochemical features of polycystic ovary syndrome in adolescents: implications for diagnosis. Hum Reprod. 2011;26:1469-77.

8. Goodman N, Cobin RH, Futterweit W, et al. Guide to the best practices in the evaluation and treatment of polycystic ovary syndrome. Endocr Pract. 2015;21:1291-300.

9. World Health Organization. Appropriate body-mass index for Asian populations and its implications for policy and intervention strategies. Geneve: WHO; 2004.

10. Gibson RS. Principles of nutritional assessment. 2nd ed. Oxford University Press; 2005. New York, New York. pp 233-42.

11. Matthews DR, Hosker JP, Rudenski AS, et al. Homeostasis model assessment: insulin resistance and beta-cell function from fasting plasma glucose and insulin concentrations in man. Diabetologia. 2000;28:2402-10.

12. Keskin M, Kurtoglu S, Kendirci M, et al. Homeostasis model assessment is more reliable than the fasting glucose/insulin ratio and quantitative insulin sensitivity check index for assessing insulin resistance among obese children and adolescents. Pediatrics. 2005;115:500-3.

13. Gutiérrez JP, Rivera-Dommarco J, Shamah-Levy T, et al. Encuesta Nacional de Salud y Nutrición 2012. Resultados nacionales. Cuernavaca, México: Instituto Nacional de Salud Pública; 2012.

14. Marsh K, Brand-Miller J. The optimal diet for women with polycystic ovary syndrome? Br J Nutr. 2005;94:154-65.

15. Norman RJ, Dewailly D, Legro RS, et al. Polycystic ovary syndrome. Lancet. 2007;370:685-97.

16. Goodarazi MO, Quiñones MJ, Azzis R, et al. Polycystic ovary syndrome in Mexican-Americans: prevalence and association with the severity of insulin resistance. Fertil Steril. 2005;84:766-9.

17. Barbieri RL, Smith S, Ryan KJ. The role of hyperinsulinemia in the pathogenesis of ovarian hyperandrogenism. Fertil Steril. 1988;50:197-212.

18. Tapia Ceballos L, López Siguero JP, Jurado Ortiz A. Prevalence of metabolic syndrome and its components in obese children and adolescent. An Pediatr. 2007;67:352-61.

19. Taylor AE. Understanding the underlying metabolic abnormalities of polycystic ovary syndrome and their implications. Am J Obstet Gynecol. 1998;179:S94-S100. 